\title{
Patientenspezifische Modellierung des passiven Bewegungsapparates als Grundlage für die präoperative Abschätzung postoperativer Folgeerscheinungen des endoprothetischen Hüftgelenkersatzes
}

\section{Patient-specific modelling of the passive musculoskeletal system as a basis for the preoperative assessment of postoperative effects of endoprosthetic hip joint replacement}

\author{
David Scherb ${ }^{1 *}$, Marlene Kurz ${ }^{1}$, Christopher Fleischmann ${ }^{2}$, Alexander Wolf ${ }^{1}$, \\ Stefan Sesselmann ${ }^{2}$, Jörg Miehling ${ }^{1}$, Sandro Wartzack ${ }^{1}$ \\ ${ }^{1}$ Engineering Design, Friedrich-Alexander-Universität Erlangen-Nürnberg \\ ${ }^{2}$ Institute for Medical Technology, Ostbayerische Technische Hochschule Amberg-Weiden \\ ${ }^{*}$ Korrespondierender Autor: \\ David Scherb \\ Martensstraße 9 \\ 91058 Erlangen \\ Telefon: +49 911/5302-96618 \\ Mail: scherb@mfk.fau.de
}

\begin{abstract}
During preoperative planning of total hip arthroplasty, the patients' biomechanics is widely reduced to geometrical parameters. To provide the orthopedist more functional biomechanical parameters, a method is shown to model a patient-specific musculoskeletal human model via CT-/MRIimages in OpenSim. Main focus of this contribution is the setup of the passive musculoskeletal system. A generic human model and a segmented dataset of bones from the hip area serve as the basis for the modelling process. During the method the generic model is processed by scaling it to the anthropometry of the patient and adjusting subject-specific kinematics and shapes gathered from the segmented data. Finally, to reach the patientspecific representation the segmented bones are integrated into the model.
\end{abstract}

\section{Keywords}

Patient-specific modeling, Image-based model, Musculoskeletal human model, OpenSim, Total Hip Arthroplasty 


\section{Motivation}

Die Produktentwicklung in der Medizintechnik stellt einen langwierigen und von einer Vielzahl von Gesetze und Verordnungen geregelten Prozess dar. Im Fokus steht dabei der Nachweis der Sicherheit und Leistungsfähigkeit des Medizinprodukts. Der Aufwand dafür ist abhängig von der Gefährdung (dem Risiko) für den Menschen. Für eine spätere Zulassung ist das Nutzen/Risiko-Verhältnis entscheidend, das bedeutet, dass bei einem hohen Risiko eines Medizinprodukts ein adäquat hoher Nutzen für den Patienten vorliegen muss [1]. Ein gutes Beispiel für ein solches Medizinprodukt ist die Hüftendoprothese. Trotz der Gefährdung des Patienten, die durch das Trennen von Muskeln, die Entfernung von Knochenmaterial und dem Einsetzen körperfremden Materials in den Organismus ausgeht, hat sich das Hüftimplantat bewährt und ist die am häufigsten implantierte Endoprothese in Deutschland mit einem weiteren erwarteten Anstieg der Implantationszahlen in den nächsten Jahren [2]. Der Nutzen des Hüftimplantats spiegelt sich am besten im Patientenwohl und den damit verbundenen Anforderungen der Patienten wider. So wird in erster Linie eine Erhöhung der Lebensqualität erwartet. Die Implantate sollen höhere Belastungen, z. B. durch sportliche Aktivitäten, über Jahre hinweg ertragen und die Bewegungsfreiheit im Gelenk nicht einschränken. Gleichzeitig soll keine Lockerung der Komponenten und möglichst wenig Abrieb bei hoher Belastung entstehen. [3]

Für den überwiegenden Anteil der Anwendungsfälle folgen daher gute Implantate, welche den Anforderungen entsprechen. Dennoch treten bei einer großen Anzahl Komplikationen bzw. Folgeprobleme auf. Diese haben ihren hauptsächlichen Ursprung im Prozess der Implantation und der damit verbundenen präoperativen Planung. Nach derzeitigem Stand werden Hüftendoprothesen vornehmlich auf Basis von zweidimensionalen Röntgenbildern geplant. Dem Arzt stehen bei der Auswahl des Implantats gewisse Implantat- und Positionsparameter zur Verfügung - beispielsweise:

- Centrum-Collum-Diaphysen-Winkel (CCD): Winkel zwischen der Femurschaftachse und der Femurhalsachse

- Antetorsionswinkel (AT): Winkel zwischen der Femurkondylenebene und der Femurhalsachse

- Hüftrotationszentrum: Punkt, um welchen das Femur rotiert wird

- Offset: Abstand zwischen der Femurschaftachse und dem Hüftrotationszentrum

- Beinlängendifferenz: Möglicher Längenunterschied der unteren Extremitäten

Aktuell wird zumeist versucht mit der Wahl dieser Parameter die Geometrie eines gesunden Gelenks so optimal wie möglich nachzubilden. Jedoch bewirkt die Rekonstruktion der skelettalen Geometrie nicht zwingend auch ein optimales Ergebnis der biomechanischen Funktion. Dies wird jedoch nicht geprüft und ist deshalb abhängig von der Erfahrung des Chirurgen. Die Folge ist daher in einigen Fällen ein aus biomechanischer Sicht funktionell schlechteres Implantat mit konsekutiv längerer und gegebenenfalls sogar unvollständiger Rehabilitation des Patienten. Dies zeigt sich $u$. a. in einem veränderten Gangbild des Patienten. Während der Standphase auf der betroffenen Seite kippt das Becken zur gegenüberliegenden Seite ab, der geschwächten Muskulatur gelingt es nicht mehr, dieses Abkippen auszugleichen und der Körper reagiert mit einer Ausgleichsbewegung des Oberkörpers. Dies ist als das Trendelenburg-Zeichen bekannt [4]. Eine Möglichkeit zur Vermeidung solcher Komplikationen ist die Erweiterung der Operationsplanung um eine muskuloskelettale Simulation, womit die postoperativen Muskelfunktionen bereits präoperativ abgeschätzt werden können. Im Folgenden wird hierzu zunächst der Stand der Forschung analysiert und darauf basierend, der Handlungsbedarf und die Forschungsziele abgeleitet. 


\section{Stand der Forschung}

\subsection{Muskuloskelettale Menschmodelle}

Muskuloskelettale Simulationen werden mit Mehrkörpersimulationsprogrammen, wie OpenSim [5], durchgeführt. Diese bieten die Möglichkeit den passiven (u. a. Knochen) und aktiven Bewegungsapparat (u. a. Muskulatur) des Menschen virtuell abzubilden. Diese Repräsentationen werden als muskuloskelettale Menschmodelle bezeichnet. Mit diesen Modellen können im Rahmen der muskuloskelettalen Simulationen körperinnere Beanspruchungen (Muskelkräfte, Gelenkmomente etc.) auf Basis von gemessenen Bewegungen berechnet werden. Diese ansonsten entweder nur indirekt oder überhaupt nicht messbaren Beanspruchungen bergen ein hohes Potential für die Verwendung in verschiedensten Bereichen. Ein möglicher Einsatz liegt in der Produktentwicklung. So ist bereits in frühen Phasen eine Ergonomiebewertung durchführbar, um das Design eines Produkts entsprechend der Ergebnisse für eine angenehmere Handhabung durch den Menschen zu optimieren [6]. Weiterhin gibt es die Möglichkeit, spezielle Produkte, wie z. B. Exoskelette, gezielt auf bestimmte Belastungen hin, auszulegen [7].

Ein weiteres, vielversprechendes Einsatzgebiet für muskuloskelettale Menschmodelle liegt im medizinischen Bereich. So ist die genauere Erforschung von Krankheiten, die einen Einfluss auf das Bewegungs- oder Interaktionsverhalten des menschlichen Körpers haben, realisierbar. Durch einen Vergleich der auftretenden Abnormalitäten mit gesunden Patienten können die Ursachen dieser Krankheiten ermittelt werden und die Auswirkungen und Effektivität möglicher Therapioptionen mittels muskuloskelettaler Simulation untersucht werden. Ein gängiges Beispiel für ein Krankheitsbild, das derzeit mit Menschmodellen erforscht wird, ist der sogenannte "Crouch Gait" [8]. Eine weitere Anwendung für muskuloskelettale Modelle im medizinischen Bereich ist die Untersuchung von Implantaten. Von großem Interesse sind hierbei Hüftimplantate. Neben der Evaluierung der resultierenden Kraft auf eine implantierte Endoprothese und der damit verbundenen Hüftkontaktkraft [9], die für die Langzeitfestigkeit des Implantats von entscheidender Rolle ist, werden mittels muskuloskelettaler Simulationen die Auswirkungen unterschiedlicher Auslegungen der zuvor erwähnten Implantats- und Positionsparameter untersucht. So ist bereits nachgewiesen, dass Variationen des Offsets [10] und des Hüftrotationszentrums [11] einen direkten Einfluss auf die Aktivierung der Muskulatur und somit auf die für den Einbeinstand nötige Muskelkraft nehmen. Damit ist gezeigt, dass der Chirurg durch die Implantation der Endoprothese die Biomechanik des Patienten unmittelbar prägen kann. Diese Wirkung sollte bereits bei der Planung berücksichtigbar sein. Die Idee ist deshalb, auf Basis der Auswahl der Implantats- und Positionsparameter eine muskuloskelettale Simulation zur Ermittlung der postoperativen muskulären Folgeerscheinungen durchzuführen.

\subsection{Patientenspezifische Modellierung}

Der entscheidende Faktor in diesem Zusammenhang ist das bei der Simulation verwendete Menschmodell. Zur Auswahl stehen dabei eine Vielzahl an frei verwendbaren OpenSourceModellen. Diese können mittels Skalierung an die Anthropometrie (Körpermaße von Menschen) des Patienten angepasst werden. Dies wird stellenweise in der Literatur bereits als patientenspezifisches Modell bezeichnet. Nicht beachtet wird dabei jedoch der individuelle Aufbau eines jeden Menschen hinsichtlich Knochenform, -geometrie, Kinematik und Muskulatur [12]. Das bedeutet, dass bei der Bestimmung der patientenindividuellen muskulären Folgen einer Hüftimplantation nur dann valide Ergebnisse erzielt werden können, wenn der zu behandelnde Patient möglichst exakt repräsentiert ist. Scheys et al. [13] unterstreicht dies durch die verbesserte Bestimmung der Länge verschiedender Momentenarme in der Hüfte. Zur Rekonstruktion der patientenindividuellen Geometrie wird ein Verfahren namens Segmentierung verwendet. Dabei ist es möglich, aus MRT-/CT-Aufnahmen 
eine 3D-Abbildung der Patienten-Knochen und -Muskulatur zu generieren [14], welche anschließend als STL-Datei abgespeichert werden. Die Segmentierung ist dabei üblicherweise ein sehr zeitaufwändiger, manueller Prozess, der abhängig von der Anzahl der zu segmentierenden Bereiche und der Bildqualität einige Tage in Anspruch nehmen kann. Nichtsdestotrotz ist damit der Aufbau eines tatsächlich patientenspezifischen Modells ausführbar. Hierfür existieren bereits Hilfsprogramme, wie der NMS Builder [15]. Dieser bietet die Möglichkeit die Knochengeometrien manuell in eine grafische Benutzschnittstelle zu laden und schrittweise nacheinander die einzelnen Gelenke, die Bewegungsmöglichkeiten der Gelenke und schließlich noch die Muskelverläufe mit Anfangs- und Endpunkten zu definieren. Das resultierende Modell kann schließlich in OpenSim exportiert und für die muskuloskelettale Simulation verwendet werden. Allerdings sind auch einige Nachteile mit dem NMS Builder im klinischen Alltag verbunden. Zunächst ist dessen komplexe Bedienung zu nennen. Chirurgen sind es gewohnt, in ihren Planungstools vereinfachte Workflows vorzufinden, die hauptsächlich automatisiert bzw. mit wenigen Klicks ablaufen. Außerdem ist die Integration von Implantaten bzw. das Einstellen der Implantat- und Positionsparameter im NMS-Builder nicht durchführbar. Dies kann erst im Nachgang mit OpenSim realisiert werden.

\section{Handlungsbedarf bzw. Forschungsproblem/-ziel}

Aus diesem Grund wird im Folgenden ein neuer Ansatz zur patientenspezifischen Modellierung vorgestellt, der automatisiert in OpenSim abläuft und bei dem auch automatisch eine Anpassung der Implantats-und Positionsparameter an die Vorgaben durch den Chirurgen realisierbar ist. Der Fokus der Modellierung liegt dabei zunächst auf dem passiven Bewegungsapparat des Patienten. Eine Besonderheit ergibt sich weiterhin durch die Beschränkung der Segmentierung auf den Beckenknochen (Pelvis) und die Oberschenkelknochen (Femora) des Patienten. Dies geschieht einerseits aufgrund der Zeitersparnis bei der Segmentierung und andererseits unter der Annahme, dass die Auswirkungen einer Hüftoperation sich nur auf diesen Bereich beschränken und deshalb die exakte Repräsentation des Patienten auch nur hier notwendig ist. Die zu beantwortende Forschungsfrage ist demnach, ob es möglich ist, ein patientenspezifisches Menschmodell auf Basis eines generischen Modells durch anthropometrische Skalierung und Intergration eines segmentierten Datensatzes zu modellieren.

\section{Material und Methoden}

\subsection{Einordnung der patientenspezifischen Modellierung in das allgemeine konzeptuelle Vorgehen}

Zum besseren Verständnis wird an dieser Stelle auf das bereits aufgestellte Konzept [16] (vgl. Bild 1) eingegangen, nach dem vorgegangen wird, um die postoperativen Folgeerscheinungen der Hüftimplantation abzuschätzen und somit dem Operateur Unterstützung bei der Operationsplanung zu geben. Die Basis bilden dabei Informationen bzw. Daten, die aus unterschiedlichen Quellen zur Verfügung stehen. Diese können in zwei verschiedene Arten aufgeteilt werden. Die Ersten sind die patientenspezifischen Informationen. Dies sind die CT/MRT-Aufnahme(n) des Hüftbereichs des Patienten, Metadaten des Patienten (Alter, Gewicht etc.), die Anthropometrie des Patienten und die Bewegungsbereiche und Muskelstärken des Patienten. Die zweite Kategorie von Informationen stellen die generischen Informationen dar. Diese bestehen aus in der Literatur bereits vorhandenen muskuloskelettalen Menschmodellen, Populationsdaten etwaiger Datenbanken und Metamodellen der Bewegung. Diese Informationen werden im Laufe des Prozesses an verschiedenen Stellen immer wieder hinzugezogen. Auf die genaue Verwendung der einzelnen Punkte wird im Folgenden nicht weiter eingegangen, kann aber anhand der Pfeile in Bild 1 nachvollzogen werden. Der erste durchgeführte Schritt ist die 
Segmentierung der CT-/MRT-Aufnahme des Hüftbereichs des Patienten. Damit werden, wie bereits oben beschrieben, die patientenspezifischen Knochen, Muskeln und Muskelansatzpunkte generiert. Diese werden zusammen mit den für jeden Muskel speziellen Parametern, die über verschiedene Methoden ermittelt werden können, in ein Modell integriert, um ein patientenspezifisches muskuloskelettales Menschmodell zu erhalten. Mit diesem Modell soll eine parametrische und biomechanische Simulation durchgeführt werden, aus der die Beeinflussung der Biomechanik bzw. Muskulatur hervorgeht. Dies kann weiter mit den 3DGeometrien der Knochen in einer Finite Elemente-Analyse genutzt werden, um zusätzlich strukturmechanische Parameter zu gewinnen. Abschließend wird basierend auf den gewonnenen Parametern dem Chirurg Feedback für seine Wahl der Implantations- und Positionsparameter der Endoprothese gegeben und womöglich in Zukunft sogar eine alternative, verbesserte Auswahl vorgeschlagen werden.

Durch die Modellierung werden demnach alle zuvor gesammelten spezifischen Informationen des Patienten in einem Menschmodell vereinigt. Weiterhin wird mit diesem Modell anschließend die muskuloskelettale Simulation durchgeführt, auf Basis derer eine veränderte Biomechanik durch die Hüftendoprothesenoperation evaluiert wird. Aus diesem Grund stellt das Gelingen der patientenspezifischen Modellierung die Grundlage für die exakte Evaluierung dar.

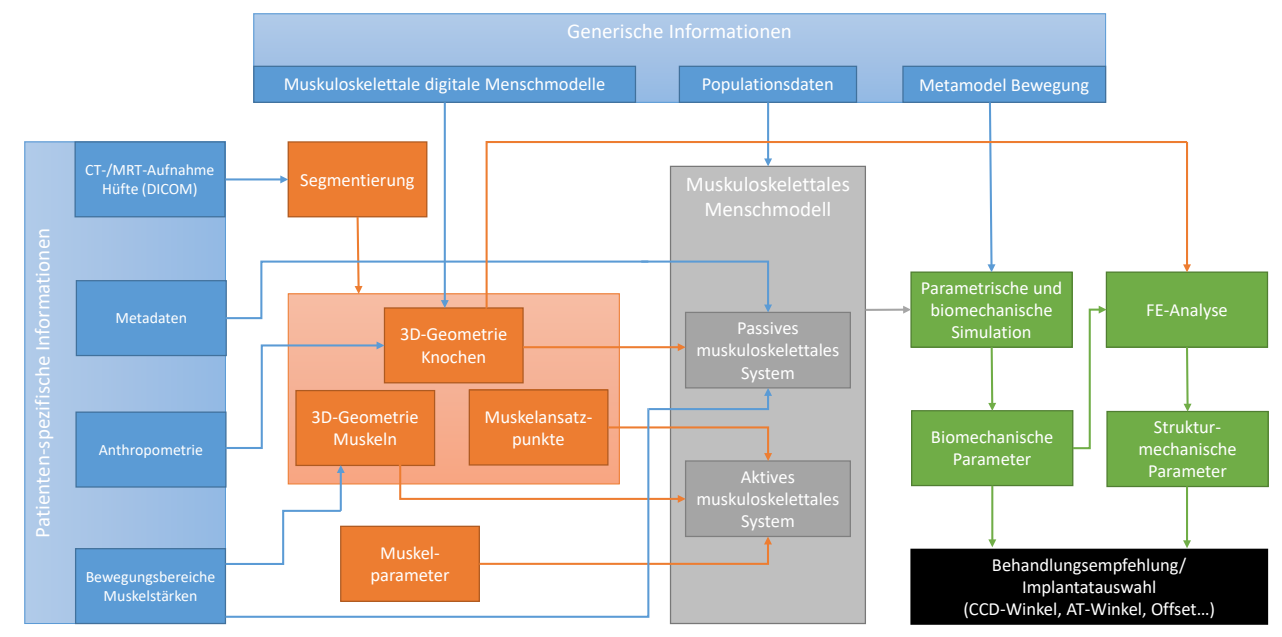

Bild 1: Aufgestellte konzeptionelle Vorgehensweise zur Ermittlung der muskuloskelettalen Folgeerscheinungen endoprothetischen Hüftersatzes nach [14]

\subsection{Vorgehensweise zur Erzeugung patientenindividueller Modelle des passiven Bewegungsapparates}

Der Kern dieses Beitrags ist die Vorstellung einer Vorgehensweise zur Generierung eines patientenspezifischen Modells des passiven Bewegungsapparates der Hüfte. Das Vorgehen ist in Bild 2 als Übersicht dargestellt. Für den Beginn wird ein generisches muskuloskelettales Menschmodell und ein segmentierter Datensatz einer Patientenhüfte benötigt. Im ersten Schritt werden die kinematischen Parameter (CCD, AT, Offset etc.) der beiden Hüften im Ausgangsmodell ermittelt. Diese werden für eine spätere Anpassung durch den Chirurgen im Rahmen der Hüftoperation benötigt. Im zweiten Schritt erfolgt eine anthropometrische Skalierung des generischen Modells an die Körpermaße des Patienten. Die Ermittlung dieser Körpermaße kann über verschiedene Wege passieren. Der Naheliegendste ist dabei, den Patienten manuell zu vermessen. Eine weitere Möglichkeit ergibt sich mit der Verwendung von empirischen Populationsdatenbanken, wie der DIN 33402-2 [17]. Diese stellt die Größenverteilung der deutschen Bevölkerung abhängig von Geschlecht und Alter dar. So kann bei fehlenden Informationen über die Körpermaße der Patient virtuell nachmodelliert bzw. abgeschätzt werden. Schließlich ist noch eine kombinierte Verwendung von beiden 
Prinzipielles Vorgehen

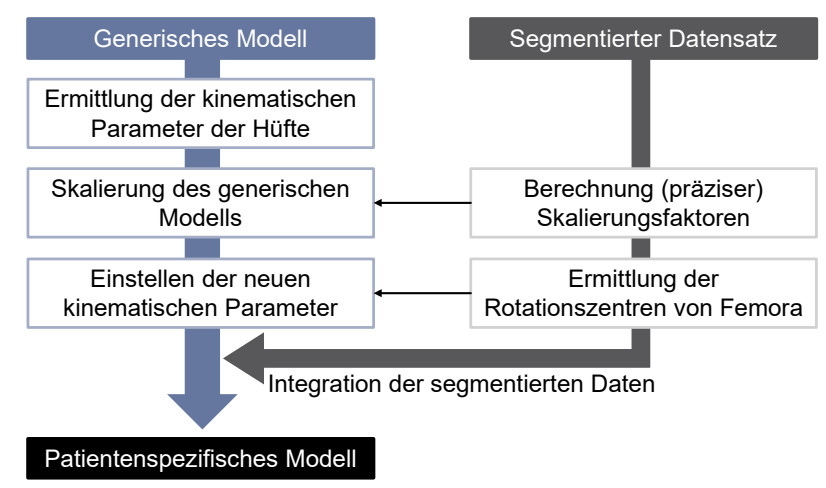

Bild 2: Prinzipeller Ablauf beim automatisierten Aufbau eines patientenspezifischen Modells

beschriebenen Möglichkeiten zur Ermittlung der Körpermaße denkbar. In jedem Fall wird die Berechnung der Skalierungsfaktoren für die Knochen des generischen Modells nach dem gleichen Muster realisiert. Diese ergeben sich durch das Verhältnis der entsprechenden Körpermaß-Distanzen, in denen der Knochen liegt: $S_{i}=\Delta L_{i, \text { Patient }} / \Delta \mathrm{L}_{i, \text { generisch; }} \mathrm{S}=$ Skalierungsfaktor, $\mathrm{L}=$ Körpermaße, $\mathrm{i}=$ betroffener Bereich/Knochen $(z w i s c h e n$ den Körpermaßen können mehrere Knochen liegen).

Da im klinischen Alltag nicht alle erforderlichen Körpermaße erfasst werden können und die Populationsdatenbank nur bestimmte Körpermaße beinhaltet, ergeben sich Abweichungen beim skalierten Modell hin zum realen Patienten. Ganz besonders kritisch ist dies an den Stellen, die patientenspezifisch modelliert werden, also vorwiegend dem Pelvis. Da hier im späteren Verlauf die exakte Repräsentation der Knochengeometrie integriert wird, ist es für die erfolgreiche Modellierung von entscheidender Bedeutung, dass der bereits vorhandene generische Knochen so gut wie möglich an die Größe des Patientenpelvis angepasst ist. Andernfalls ergeben sich durch die Integration Veränderungen bei den Größenabmessungen, wodurch das muskuloskelettale Modell nicht mehr mit dem Patienten übereinstimmt. Damit wäre auch die korrekte Abschätzung einer veränderten Biomechanik nach dem Hüftendoprothesenersatz nicht mehr zu garantieren. Aus diesem Grund wird für die Berechnung der Skalierungsfaktoren des generischen Pelvis der bereits segmentierte Pelvis des Patienten herangezogen und durch dessen Verwendung, der Skalierungsfaktor in jede Raumrichtung berechnet. Dazu werden sogenannte anatomische Landmarken benutzt. Dies sind definierte Bereiche/Punkte an den Knochen. Folglich werden die äquivalenten anatomischen Landmarken am generischen und segmentierten Pelvis, zu sehen in Bild 3, manuell identifiziert. Anschließend werden die Distanzen der einzelnen Landmarken zueinander berechnet und als Vektor abgespeichert. Die Skalierungsfaktoren berechnen sich schließlich über das Verhältnis der jeweiligen Distanzen in generischen und segmentierten Pelvis. Bei Beachtung aller Landmarken erfolgt jedoch eine Skalierung nach der Form des segmentierten Pelvis und nicht nach dessen Größe. Deswegen werden nur vier Landmarken (auf einer Pelvishälfte) verwendet, die ungefähr die äußersten Abmessungen des Pelvis darstellen: anterior superiot iliac spine (ASIS), posterior superior iliac spine (PSIS), upper iliac crest und iliac ischiac tuberosity. Diese sind in Bild 3 markiert. Der Skalierungsfaktor in XRichtung ergibt sich dementsprechend aus dem Verhältnis der sagittalen Distanz zwischen ASIS und PSIS von Patientenpelvis zu generischem Pelvis (jeweils der Mittelwert aus linker und rechter Hälfte). Der y-Skalierungsfaktor wird durch die entsprechende Raumdistanz zwischen upper iliac crest und iliac ischiac tuberosity (ebenfalls Mittelwert zwischen linker und rechter Hälfte) gebildet und der z-Skalierungsfaktor wird durch den Abstand der beiden ASISLandmarken berechnet. Mit dem gleichen Verfahren könnte eine präzise Skalierung der Femora mit entsprechenden anatomischen Landmarken durchgeführt werden. Im Falle des nachfolgend vorgestellten Demonstrators wird darauf allerdings aufgrund eines mangelhaften 
Datensatzes verzichtet und die Femora entsprechend durch anthropometrische Skalierung angepasst.

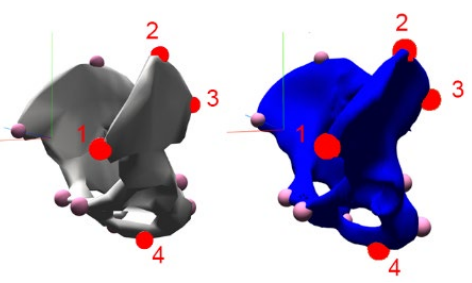

Bild 3: Generischer Pelvis (weiß) und segmentierter Patientenpelvis (blau) mit äquivalenten anatomischen Landmarken (pink); rot markiert sind die für die Pelvis-Skalierung verwendeten Landmarken: 1 anterior superior iliac spine (ASIS), 2 upper iliac crest, 3 posterior superior iliac spine (PSIS), 4 iliac ischiac tuberosity

Weiterhin ist es wichtig zu erwähnen, dass dem segmentierten Pelvis bei dem Vorgang der Segmentierung ein lokales Koordinatensystem (KOS) aufgeprägt wird. Dieses weicht von dem loaklem OpenSim-KOS ab, was zur falschen Berechnung der Skalierungsfaktoren des Pelvis und einer inkorrekten Integration des segmentierten Pelvis in das Modell führens würde. Deswegen wird in dem segmentierten Pelvis ein neues KOS gemäß den Vorgaben der International Society of Biomechanics (IBS) [18] definiert. Die IBS empfiehlt dafür erneut die Verwendung der anatomischen Landmarken. So wird zuerst eine Linie zur Verbindung der beiden ASIS-Landmarken gezogen. Diese definiert die erste Achse des neuen KOS. Im nächsten Schritt wird eine Lotgerade zu einer Verbindungslinie der beiden PSIS-Landmarken gefällt. Diese repräsentiert die zweite Achse des neu definierten KOS. Die dritte Achse ergibt sich schließlich durch das Kreuzprodukt der beiden vorhandenen Achsen. Abschließend wird eine Transformationsmatrix, bestehend aus einer Rotationsmatrix und der Translation des Ursprungs, für die Umrechnung in das OpenSim-KOS aufgestellt.

Im Anschluss an die Skalierung des generischen Modells werden die kinematischen Parameter der beiden Hüftgelenke entsprechend denen des Patienten eingestellt. Dazu erfolgt zuerst die Bestimmung der beiden Hüftrotationszentren. Dafür wird wieder der segmentierte Datensatz des Patienten verwendet. Für die Bestimmung existieren verschiedene Methoden [19]. Die Wahl fällt auf die fit sphere-Methode, da diese einfach zu implementieren ist und mit dieser das Hüftrotationszentrum mit hoher Genauigkeit bestimmt werden kann. Bei der Methode wird mittels Optimierung eine Kugel in den Femurkopf des segmentierten Femurs gelegt, dessen Mittelpunkt das Hüftrotationszentrum darstellt. Die Defintion des Hüftgelenks im generischen Modell wird anschließend entsprechend den neu berechneten Hüftrotationszentren durch Translation der Femora angepasst. Auf Basis dessen und der zu Beginn ermittelten kinematischen Parameter können schließlich in Zukunft die kinematischen Variationen durch den Chirurgen im Zuge der Planung der Endoprothese mittels Translation/Rotation des betreffenden Femurs nachgebildet werden.

Den letzten Schritt hin zum patientenspezifischen Modell stellt die Integration der segmentierten Daten in das skalierte Modell zur Nachbildung der relevanten skelettalen Anatomie des Patienten dar. Dabei wird die zuvor aufgestellte Transformationsmatrix benutzt, aus der sich die notwendigen Rotationswinkel um die drei Hauptachsen und die Translation des Ursprungs berechnen lassen.

\subsection{Anwendung der dargestellten Vorgehensweise}

Das vorgestellte Vorgehen wurde anhand von Beispieldaten überprüft. Als Ausgangsmodell wurde ein Ganzkörpermodell von Miehling [20] verwendet. Der segmentierte Datensatz stammte von einer Frau. Der Scan der Femora war dabei nicht komplett. Sonst lagen keine weiteren Informationen bzgl. der Patientin vor. Aus diesem Grund wurde über die 
Abmessungen des segmentierten Datensatzes die Körpergröße der Frau abgeschätzt. Mittels der DIN 33402-2 wurden darauf aufbauend weitere Körpermaße bestimmt. Alle Körpermaße der virtuellen Patientin sind in Tabelle 1 dargestellt. Eine weitere Besonderheit lag bei der Definition des neuen KOS des segmentierten Pelvis. Dieses wurde auf den Mittelpunkt zwischen den beiden ASIS gesetzt und zusätzlich mit 13,6 um die Achse zwischen Ursprung und ASIS rotiert. Dies geschah gemäß der äquivalenten Definition des KOS im Ausgangsmodell. Weiterhin wurde für die fitSphere-Methode zur Bestimmung der Hüftrotationszentren der gleichnamige Algorithmus von Matlab (2016, MathWorks) verwendet. Das restliche Vorgehen entsprach der zuvor beschriebenen Vorgehensweise.

Tabelle 1: Körpermaße der virtuellen Patientin

\begin{tabular}{ll}
\hline Körpermaß & Wert \\
\hline Masse & $63,8 \mathrm{~kg}$ \\
Körpergröße & $1,57 \mathrm{~m}$ \\
Schulterhöhe & $1,30 \mathrm{~m}$ \\
Hüfthöhe & $0,96 \mathrm{~m}$ \\
Schrittlänge & $0,75 \mathrm{~m}$ \\
Fingerhöhe & $0,63 \mathrm{~m}$ \\
Kniehöhe & $0,41 \mathrm{~m}$ \\
Fußbreite & $0,09 \mathrm{~m}$ \\
Fußlänge & $0,24 \mathrm{~m}$ \\
\hline
\end{tabular}

\section{Ergebnisse und Diskussion}

Zur Beurteilung der erfolgreich durchgeführten Transformation des generischen Modells in ein patientenspezifisches Modell wird zuerst die Skalierung hinsichtlich der Anthropometrie verifiziert. Dafür wurden die Körpermaße am skalierten Modell bestimmt und mit den jeweiligen Vorgaben des virtuellen Patienten verglichen. Speziell wurde jeweils die linke und rechte Körperhälfte verglichen, um etwaige Abweichungen auszuschließen. Bild 4a zeigt dazu die prozentuelle Abweichung. So ist zu erkennen, dass die meisten skalierten Körpermaße weniger als $0,3 \%$ (numerisch nicht mehr als $5 \mathrm{~mm}$ ) von den Vorgaben abweichen. Die Masse, Fußbreite und -länge stimmen nach der Skalierung sogar genau mit den Patientendaten überein. Die größte Abweichung tritt bei der Hüfthohe auf. Dies lässt sich auf die derzeitige Definition der Hüfthöhe zurückführen. Die Hüfthöhe ist als solche nicht in der DIN 33402-2 aufgeführt, deswegen wird sie zur Skalierung durch die Schritthöhe plus den vertikalen Abstand zwischen iliac ischiac tuberosity und ASIS auf der jeweiligen Seite vorgegeben. Dabei wird einerseits angenommen, dass die Schritthöhe und die iliac ischiac tuberosity auf der gleichen Höhe sind, was nicht zwangsläufig der Fall ist und andererseits wird der Skalierungsfaktor bei der Schrittlänge mit in die Gleichung übernommen. So kann die im Vergleich zum Rest große Abweichung der Hüfthöhe erklärt werden. Dies sollte bei einer späteren Skalierung des Modells nach realen Patienten durch Messung der ASIS-Höhen als Vorgabe für die Hüfthöhe noch validiert werden.

Zur weiteren Beurteilung der erfolgreichen patientenspezifischen Modellierung wird die Integration des segmentierten Datensatzes in das skalierte Modell beurteilt, die in Bild 4b zu sehen ist. Dabei zeigt sich, dass die Ebene zwischen den ASIS und PSIS der beiden Pelves übereinstimmt, wodurch das KOS identisch sein sollte und somit der segmentierte Pelvis an der richtigen Stelle eingefügt ist. Außerdem stimmen die ASIS- Abstände und die Distanzen zwischen ASIS und PSIS überein. Dadurch ist auch die Skalierung des Pelvis in Breite und Tiefe korrekt. Abweichungen könnten in der Höhe vermutet werden. Allerdings sind diese auf 
die unterschiedliche Form der beiden Pelves zurückführen, die sehr gut am oberen Kamm und im unteren Bereich des Pelvis zu erkennen sind. Der segmentierte Pelvis ist entsprechend nach optischer Begutachtung korrekt in das skalierte Modell integriert.

Im Laufe der Modellierung werden jedoch einige Ungenauigkeiten bzw. Annahmen zur Vereinfachung hingenommen. Die Erste ist die Bestimmung der Hüftrotationszentren des Patienten. Durch die fit sphere-Methode ist keine exakte Ermittlung des Hüftrotationszentrums möglich, die Abschätzung weicht immer etwas vom realen Punkt ab. [19] Dies liegt an der Tatsache, das versucht wird, eine Kugel in den Femurkopf zu legen. Dieser besitzt jedoch bei keinem Menschen die exakte Form einer Kugel. Die Form ähnelt eher einem Ellipsoid. Des Weiteren liefert der verwendete Algorithmus keine reproduzierbaren Ergebnisse. Das Resultat am gleichen Femur-Datensatz ist bei jedem Durchlauf verschieden, sodass sich Variationen von ein paar Millimeter in jede Raumrichtung ergeben. Ein weiterer zu beachtender Faktor ist die Bestimmung der Landmarkenpositionen. Diese sind teilweise nicht exakt definiert, bzw. beschreiben ganze Bereiche und keine einzelnen Punkte der knöchernen Struktur. Somit sind Abweichungen bei der manuellen Lokalisation nicht auszuschließen, die sich unmittelbar auf die Pelvisskalierung und Bestimmung des KOS auswirken. Aus diesem Grund wäre die Verwendung eines automatischen Determinationsalgorithmus für das Erzielen reproduzierbarer Ergebnisse zu empfehlen. Die letzte getroffene Annahme ist, dass die Femora und der Pelvis des segmentierten Patienten die gleiche Orientierung besitzen. Dies ist jedoch abhängig von der Positionierung des Patienten bei der Aufnahme. Zur Überprüfung müsste ähnlich wie beim Pelvis ein KOS in den Femora durch Landmarken definiert werden. Dazu wird allerdings das komplette Femur benötigt. Deswegen musste in diesem Falle die gleiche Orientierung angenommen werden.

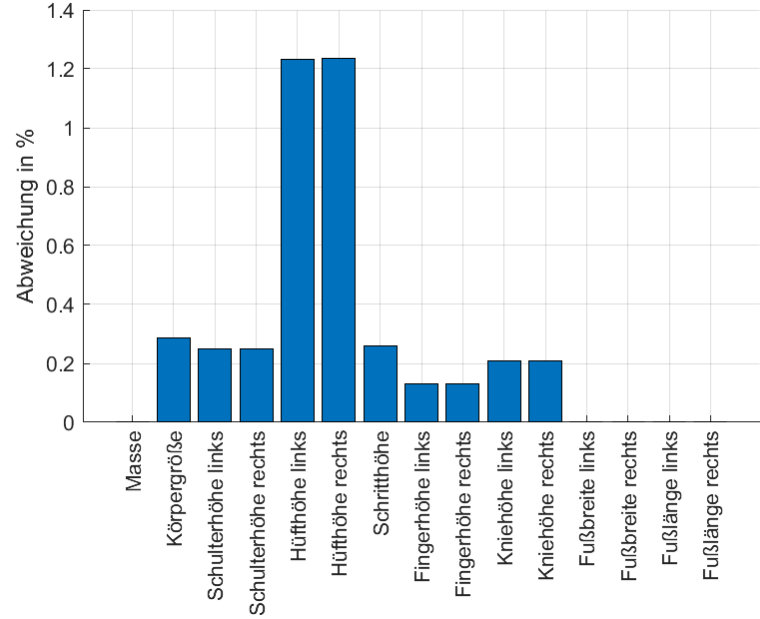

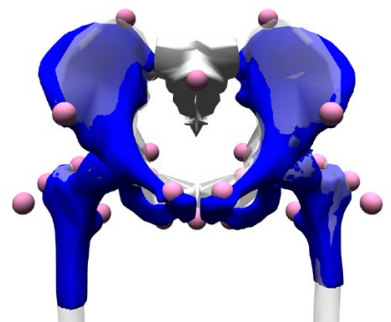

b

Bild 4: a) Prozentuelle Abweichung der Anthropometrie des skalierten generischen Modells zu den vorgegebenen virtuell erzeugten Patienten, b) Integration der segmentierten Daten (blau) in das skalierte generische Modell (weiß/transparent)

\section{Zusammenfassung und Ausblick}

Resümierend kann festgehalten werden, dass mittels der vorgestellten Methode die Integration eines segmentierten Datensatzes in ein generisches Modell zur Generierung eines patientenspezifsichen Modells des passiven Bewegungsapparates fast automatisiert ermöglicht wird. Der einzige Eingriffspunkt durch den Anwender ist derzeit noch die Bestimmung der Landmarken. Darauf aufbauend kann in Zukunft der muskuläre Bereich der Hüfte mit den spezifischen Muskelansatzpunkten und -pfaden des Patienten in das Modell eingefügt werden, wodurch das patientenspezifische muskuloskelettale Modell vollständig 
modelliert wäre. Die resultierenden Modelle müssen anschließend noch mit realen Patientendaten verglichen werden, um eine Validierung der Modellierung zu erzielen.

\section{Danksagung}

Diese Forschungsarbeit ist Teil des Projekts "Kompetenz- und Analyseprojekt für die datengetriebene Prozess- und Produktionsoptimierung mittels Data Mining und Big Data (E|ASY-Opt)" und wird vom Bayerischen Programm "Investitionen in Wachstum und Beschäftigung" vom Europäischen Fonds für regionale Entwicklung (ERDF, 2014-2020) gefördert. Es wird vom Bayerischen Staatsministerium für Wirtschaft, Landesentwicklung und Energie verwaltet. Die Autoren sind verantwortlich für den Inhalt dieser Veröffentlichung.

\section{Literaturverzeichnis}

[1] Bundesverband Medizintechnologie: Der lange Weg eines Medizinprodukts von der Idee bis zur Anwendung am Patienten. URL https://www.bvmed.de/de/bvmed/publikationen/medizinprodukte-inforeihe Überprüfungsdatum 2020-06-03

[2] Grimberg, Alexander ; Jansson, Volkmar ; Melsheimer, Oliver: Jahresbericht 2019. Berlin : EPRD Deutsche Endoprothesenregister, 2019

[3] Learmonth, Ian D. ; Young, Claire ; Rorabeck, Cecil: The operation of the century: total hip replacement. In: The Lancet 370 (2007), Nr. 9597, S. 1508-1519

[4] Madsen, Michael S. et al.: The effect of total hip arthroplasty surgical approach on gait. In: Journal of Orthopaedic Research 22 (2004), Nr. 1, S. 44-50

[5] Delp, Scott L. et al.: OpenSim: open-source software to create and analyze dynamic simulations of movement. In: IEEE transactions on bio-medical engineering 54 (2007), Nr. 11, S. 1940-1950

[6] Wolf, Alexander et al.: Towards Virtual Assessment of Human Factors: A Concept for Data Driven Prediction and Analysis of Physical User-product Interactions. In: Proceedings of the Design Society: International Conference on Engineering Design 1 (2019), Nr. 1, S. 4029-4038

[7] Miehling, Joerg ; Wolf, Alexander ; Wartzack, Sandro: Musculoskeletal Simulation and Evaluation of Support System Designs. In: Karafillidis, Athanasios; Weidner, Robert (Hrsg.): Developing Support Technologies Integrating Multiple Perspectives to Create Assistance that People Really Want : Springer, 2018, 219-227

[8] Arnold, Allison S. et al.: The role of estimating muscle-tendon lengths and velocities of the hamstrings in the evaluation and treatment of crouch gait. In: Gait \& posture 23 (2006), Nr. 3, S. 273-281

[9] Lenaerts, G. et al.: Aberrant pelvis and hip kinematics impair hip loading before and after total hip replacement. In: Gait \& posture 30 (2009), Nr. 3, S. 296-302

[10] Miehling, Jörg et al.: Biomechanischer Simulator zur prä-operativen Abschätzung und Optimierung der postoperativen Muskelfunktion am Beispiel der Revisionsendoprothetik des Hüftgelenks. Deutscher Kongress für Orthopädie und Unfallchirurgie (DKOU), Berlin, 2016

[11] Delp, Scott L. ; Maloney, William: Effects of hip center location on the moment-generating capacity of the muscles. In: Journal of biomechanics 26 (1993), 4-5, S. 485-499

[12] Fleischmann, Christopher et al.: Femoral Shape and Size Variability from segmented CT datasets for patientspecific THA planning. In: Current Directions in Biomedical Engineering (angenommen)

[13] Scheys, Lennart et al.: Personalized MR-based musculoskeletal models compared to rescaled generic models in the presence of increased femoral anteversion: effect on hip moment arm lengths. In: Gait \& posture 28 (2008), Nr. 3, S. 358-365

[14] Fleischmann, Christopher et al.: Segmentation of musculotendinous structures of the hip from 3D imaging for patient-specific individualization of biomechanical simulations. In: Proceedings of the 3rd International Conference on Human Interaction and Emerging Technologies (IHIET 2020), August 27-29, Paris, France

[15] Valente, Giordano et al.: nmsBuilder: Freeware to create subject-specific musculoskeletal models for OpenSim. In: Computer methods and programs in biomedicine 152 (2017), S. 85-92

[16] Scherb, David et al.: Conceptual Approach to estimate the musculoskeletal outcome of endoprosthetic hip replacements. CAMS Knee OpenSim-Workshop, ETH Zürich, 2020

[17] DIN 33402-2. 2005. Ergonomie-Körpermaße des Menschen-Teil 2: Werte

[18] Wu, Ge et al.: ISB recommendation on definitions of joint coordinate system of various joints for the reporting of human joint motion-part I: ankle, hip, and spine. In: Journal of biomechanics 35 (2002), Nr. 4, S. 543-548

[19] Ehrig, Rainald M. et al.: A survey of formal methods for determining the centre of rotation of ball joints. In: Journal of biomechanics 39 (2006), Nr. 15, S. 2798-2809

[20] Miehling, Jörg: Musculoskeletal modeling of user groups for virtual product and process development. In: Computer methods in biomechanics and biomedical engineering 22 (2019), Nr. 15, S. 1209-1218 